FRI0316

THE PRESENCE OF ANTI-RITUXIMAB ANTIBODIES PREDICTS INFUSION-RELATED REACTIONS IN PATIENTS WITH SYSTEMIC LUPUS ERYTHEMATOUS

C. Wincup, M. Menon, E. Smith, E.C. Jury, D.A. Isenberg, C. Maurion behalf of ABIRISK Consortium. Rheumatology, University College London, London, UK

Background: Rituximab is a genetically engineered chimeric anti-CD20 monoclonal antibody commonly used in the treatment of a variety of autoimmune rheumatic conditions. Although generally well tolerated, serious infusion related reactions can occur and are difficult to predict. It has previously been suggested that patients with systemic lupus erythematosus (SLE) who are treated with Rituximab demonstrate a higher incidence of infusion reactions and a less predictable response to the therapy when compared with other diseases.

A major limitation of biologic therapies is the development of anti-drug antibodies (ADA). To date the presence of ADAs to Rituximab have not previously been explored in detail.

Objectives: To assess the prevalence of ADAs to Rituximab in patients receiving treatment for SLE and how the presence of these antibodies relates to:

1. the incidence of infusion-related reactions

2. the therapeutic efficacy of the drug

Methods: We assessed the presence of Rituximab ADAs using a Meso Scale Discovery platform in 58 SLE patients treated with Rituximab at University College London Hospital, UK. A subgroup of 42 patients were followed up longitudinally for up to 8 years after their first dose of Rituximab. Clinical parameters including BILAG, complement C3 levels, anti-double-stranded DNA (dsDNA) antibody titres, lymphocyte count and frequency of $\mathrm{CD} 19^{+} \mathrm{B}$ cells were recorded. A retrospective review of patient notes was undertaken to assess for a history of infusion related reactions. Mann-Witney $U$ test was used to compare variables between the ADA positive group and ADA negative group.

Results: A total of 58 patients were recruited of which $88 \%$ were female $(51 / 58)$ Median age was 38.9 years old with a median disease duration of 84 months ADAs to Rituximab were detected in $60 \%$ (35/58) of patients. In those with a positive ADA ( $n=35), 22$ were later retreated with Rituximab. Infusion reactions were noted in $68 \%(15 / 22)$ of those undergoing retreatment in the context of a previously positive ADA. In the remaining patients who were retreated but in which no infusion reaction was seen (7/22), the average level of ADA was significantly lower than in those who did have a reaction (median in those who suffered a reaction=200 unit; median in those who were ADA positive but did not develop a reaction on retreatment $=10$ units; $p=0.008$ ). There were no reactions observed in patients that did not display ADAs who were subsequently retreated.

In the first six months post-Rituximab there was no statistically significant difference in BILAG, C3 levels, dsDNA titres, lymphocyte count or CD19 between the groups in which ADAs were present when compared with those in which ADAs were absent.

Conclusions: For the first time, ADAs to Rituximab have been described in a cohort of SLE patients who have undergone B-cell depletion therapy. High levels of circulating ADAs to Rituximab were found in patients who later developed infusion reactions on retreatment. However, there was no obvious difference in clinical outcomes between those who were ADA positive compared with those in which it was negative. Together, this suggests the presence of ADAs can predict future infusion reactions but do not adversely impact upon the efficacy of treatment.

In future, the routine screening of ADAs to Rituximab will help to reduce the risk of serious infusion reactions in patients being treated.

Disclosure of Interest: None declared

DOI: 10.1136/annrheumdis-2018-eular.3644

\section{FRI0317 REASONS FOR HOSPITALISATION AND IMMUNOSUPPRESSANT USE IN ADULT PATIENTS WITH SYSTEMIC LUPUS ERYTHEMATOSUS ADMITTED TO A TERTIARY ACADEMIC MEDICAL CENTRE}

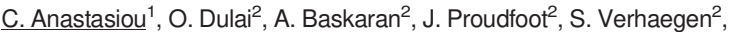
K. Kalunian ${ }^{2}$. University of California San Diego and University of California San Francisco; ${ }^{2}$ University of California San Diego, San Diego, USA

Background: An estimated $22 \%$ to $28 \%$ of US SLE patients are hospitalised each year. ${ }^{1}$ SLE and other connective tissue diseases have been ranked the sixth most likely conditions to result in 30 day readmission to the hospital. ${ }^{2}$

Objectives: To determine if the number of SLE patient hospital admissions, primary reasons for hospitalisation, and mortality have changed over time. We also sought to evaluate changes in immunosuppressant use in these patients.

Methods: A retrospective chart review was conducted on all adult patients with SLE admitted to the University of California San Diego Medical Centre in 2005 and 2013. Patients were identified by SLE billing code 710.0 of the International Classification of Diseases, Ninth Revision, Clinical Modification (ICD-9-CM).

Results: The number of yearly SLE patient hospitalizations decreased from 178 to 86 between the two times of observation. Infection was the most common reason for hospitalisation accounting for $39.9 \%$ of hospitalizations in 2005 versus $31.4 \%$ of hospitalizations in 2013 ( $p=0.29$ ). Lupus flare accounted for $9.6 \%$ of admissions in 2005 versus $8.1 \%$ of admissions in 2013 ( $p=0.722)$. SLE patient mean length of hospital stay decreased from 7.6 days to 6.4 days compared to all patient length of stay which decreased from 6 days to 5.75 days. Seven patients (3.9\%) died during their hospitalisation in 2005 as opposed no inpatient deaths in 2013. Of the 261 admissions between 2010 and 2013, six admissions resulted in death (2.3\%). Corticosteroid use decreased $(79.8 \%$ to $61.6 \%, p=0.109$ ), while hydroxychloroquine $(27.0 \%$ to $59.3 \%$, $\mathrm{p}<0.001)$, mycophenolate mofetil $(5.6 \%$ to $13 \%, p=0.060)$, and leflunomide $(0.6 \%$ to $9.3 \%, p=0.008)$ use increased over time.

Conclusions: Hydroxychloroquine and leflunomide use in hospitalised patients more than doubled from 2005 to 2013 with statistical significance. Rise in myco phenolate mofetil use and drop in corticosteroid use trended toward statistical significance. Number of hospitalizations, length of stay, and mortality decreased Infection was the primary reason for inpatient hospitalisation and there was a decrease in admission for both infections and SLE flares between the time points although this did not reach statistical significance. We hypothesise that changing medication prescribing patterns may have altered the rate of damage accrual and mortality in SLE patients, and plan to perform a national study to further evaluate SLE hospitalisation data on a larger scale.

\section{REFERENCES:}

[1] Chakravarty EF, Bush TM, Manzi S, et al. Prevalence of adult systemic lupus erythematosus in California and Pennsylvania in 2000: estimates obtained using hospitalization data. Arthritis Rheum 2007;56(6):20922094.

[2] Elixhauser A, Steiner C. Readmissions to U.S. Hospitals by Diagnosis, 2010: Statistical Brief \#153. Healthcare Cost and Utilization Project (HCUP) Statistical Briefs. Agency for Healthcare Policy and Research. URL: http://www.ncbi.nlm.nih.gov/books/NBK52651/ [Accessed May 2016].

Disclosure of Interest: None declared

DOI: 10.1136/annrheumdis-2018-eular.4228

\section{FRI0318 HEALTH CARE RESOURCE UTILISATION (HCRU) AND COST ANALYSES OF SYSTEMIC LUPUS ERYTHEMATOSUS (SLE) AS A FUNCTION OF DISEASE SEVERITY: ANALYSIS OF REAL-WORLD CLAIMS DATA FROM A GERMAN SICKNESS FUND}

E.R. Hammond ${ }^{1}$, H. Friedel ${ }^{2}$, E. Garal-Pantaler ${ }^{2}$, M. Pignot ${ }^{3}$, E. Velthuis ${ }^{4}$ X. Wang ${ }^{1}, \mathrm{H} . \mathrm{Nab}^{5}$, B. Desta ${ }^{1}$, A. Schwarting ${ }^{6} .{ }^{1}$ AstraZeneca, Gaithersburg, MD, USA; ${ }^{2}$ Team Gesundheit GmbH, Essen, Germany; ${ }^{3}$ Kantar Health GmbH, Munich, Germany; ${ }^{4}$ Evidera PPD, Utrecht Area, Netherlands; ${ }^{5}$ AstraZeneca, Cambridge, UK: ${ }^{6}$ Universitätsmedizin der Johannes Gutenberg-Universität Mainz, Mainz, Germany

Background: SLE is a severe, chronic autoimmune disease of the connective tis sue involving multiple organ systems. Understanding the economic burden of SLE in the context of disease severity is important when considering new therapeutic options.

Objectives: HCRU and costs associated with SLE were examined retrospectively using anonymized data from a German Sickness Fund database.

Methods: Real-world claims for adult ( $\geq 18$ years old) patients ( $p t s)$ with SLE from a German Sickness Fund database of company health insurance schemes were analysed. HCRU and costs were assessed annually for 2009-2014 for pts diag nosed with ${ }^{\text {SLE in } 2009}$ and validated using repeated SLE-related claims, co-diagnosis codes, laboratory tests, prescription treatment, and the diagnosing physician's specialty. Pts had to have data available for 2009 and $\geq 3$ years before the index quarter in 2009. HCRU and costs for SLE cases were compared with those of controls matched $(4: 1)$ by age, sex, and baseline Charlson Comorbidity Index (CCI). Continuous outcomes were compared with a nonparametric test (e.g., WilcoxonMann-Whitney) because most outcome distributions were positively skewed.

Results: Of the 3,290,701 persons with data available for 2009 and $\geq 3$ years prior, 1228 had an SLE diagnosis in 2009. SLE prevalence steadily increased from $37.32 / 100,000$ (incidence: 5.96/100,000 per year) in 2009 to $47.36 / 100,000$ in 2014. The final sample comprised 1,160 SLE-confirmed pts (mean age: 52 years; females: $84 \%$; baseline $\mathrm{CCI}$ range: $1-13)$. Most (85\%) pts were diagnosed with SLE before 2009 SLE disease severity at baseline was classified as mild for 148 , moderate for 484 , and severe for 528 pts using a combination of International Classification of Diseases-10 GM and medication/procedures codes. Compared with matched controls, SLE pts, overall and those with moderate and severe disease, had significantly greater mean annual medical costs in 2009 (all SLE: $€ 6895$ vs $€ 3,692$; moderate SLE: $€ 4867$ vs. $€ 3,380$; severe SLE: $€ 10001$ vs. $€ 4,239 ; \mathrm{p}<0.0001$ for each comparison) and each year thereafter. Mean costs, total number of hospital days, numbers of outpatient visits, hospital stays, and outpatient prescriptions, and other benefits were significantly greater for all 
pts with SLE and for those with moderate and severe disease vs. matched controls. For example, for pts with severe SLE vs. controls, mean costs for hospital stays, outpatient prescriptions, and other benefits were $€ 4335$ vs. $€ 1,414$, $€ 2582$ vs. $€ 1,087$, and $€ 1068$ vs. $€ 691$, respectively, in 2009.

Conclusions: In Germany, the economic burden of moderate and severe SLE was greater than that of sociodemographic- and morbidity-adjusted controls between 2009 and 2014. Pts with SLE incurred greater HCRU and total annual medical costs vs. matched controls. HCRU increased with increasing SLE disease severity, with the greatest burden among pts with severe disease. New treatments could reduce HCRU and future costs.

Disclosure of Interest: E. Hammond Employee of: AstraZeneca, H. Friedel: None declared, E. Garal-Pantaler: None declared, M. Pignot: None declared, E. Velthuis: None declared, X. Wang Employee of: AstraZeneca, H. Nab Employee of: AstraZeneca, B. Desta Employee of: AstraZeneca, A. Schwarting: None declared DOI: 10.1136/annrheumdis-2018-eular.5150

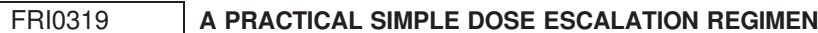 FOR MILD HYDROXYCHLOROQUINE-INDUCED HYPERSENSITIVITY REACTION}

E. Takamasu, Y. Nasa, H. Takahashi, S. Sugii, N. Yokogawa. rheumatology, Tokyo metropolitan tama medical center, Tokyo, Japan

Background: In a recent Japanese clinical trial of hydroxychloroquine (HCQ), HCQ-induced hypersensitivity reaction occured in 4.9\% (5/103) including a case of Stevens-Johnson syndrome. ${ }^{1}$ Several desensitisation methods were proposed, however the protocols were very complicated for routine practice. ${ }^{2}$ Recently, a simple dose-escalation regimen of sulfamethoxazole-trimethoprim (SMX-TMP) showed better tolerability than standard dosing. ${ }^{3}$

Objectives: To investigate the incidence and risk factors of HCQ-induced hypersensitivity and propose a simple dose-escalation regimen for HCQ-induced hypersensitivity.

Methods: This is a single-centre, retrospective study. The subjects were Japanese SLE patients who started HCQ between Apr 1, 2009 and June 30, 2017 at Tokyo Metropolitan Tama Medical Centre. HCQ was prescribed with a dosage of $6.5 \mathrm{mg} / \mathrm{ideal}$ body weight (IBW) $\mathrm{kg}$ or less. We identified the cases of HCQinduced hypersensitivity by reviewing electronic medical charts and pictures taken by patients or physicians. We diagnosed with HCQ-induced hypersensitivity if generalised eruption occurred within a month of starting $\mathrm{HCQ}$ and resolved completely after withdrawal of HCQ. We used the simple dose escalation regimen, starting from $40 \mathrm{mg} /$ day with weekly increments by $40 \mathrm{mg}$, for patients with HCQinduced hypersensitivity who did not require hospitalisation or systemic administration of glucocorticoids. We compared the clinical parameters (age, sex, dosage/actual or ideal body weight, starting dose, use of immunosuppresives, use of SMX-TMP, profile of autoantibodies) between patients with HCQ-induced hypersensitivity and those without HCQ-induced hypersensitivity. We also evaluated the success rate of our dose escalation regimen.

Results: Total 234 SLE patients were analysed. HCQ-induced hypersensitivity reaction were identified in 20 cases $(8.5 \%)$. All cases developed mild generalised maculopapular rashes 17 (IQR 7.8-28.8) days after initiation of HCQ. No patient required hospitalisation or systemic glucocorticoid therapy. Among the clinical parameters compared, only anti-Sm antibody was associated with HCQ-induced hypersensitivity (table 1). HCQ was restarted in 10 patients using the simple dose escalation regimen. Among them 9 patients successfully continued HCQ and only one discontinued due to reappearance of mild hypersensitivity reaction.

\begin{tabular}{|c|c|c|c|}
\hline & 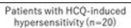 & 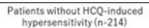 & $\begin{array}{c}\text { Pvalue } \\
\text { Pand }\end{array}$ \\
\hline Agec years (liper) & $38(28.46)$ & $40(30-52)$ & 0.490 \\
\hline Femate. $x$ & 85.0 & 93.5 & 0.163 \\
\hline Body weight, kg (IIQR) & $50(45-55)$ & $52(47-60)$ & 0.178 \\
\hline 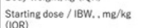 & 5.1 (4.0.6.60) & 5.3(45.5.59) & 0.620 \\
\hline BMM (IQR) & $20.3(1822 \cdot 21.8)$ & $20.8(18.9 \cdot 23.3)$ & 0.241 \\
\hline \multicolumn{4}{|l|}{ HCQ stating dose } \\
\hline -100me \& & 5.0 & 0.5 & \\
\hline$-150 \mathrm{mg}$ & 0 & 0.5 & \\
\hline$-200 \mathrm{me} \%$ & 45.0 & 430 & \\
\hline$-300 \mathrm{me} \times$ & 45.0 & 51.4 & \\
\hline$-400 \mathrm{me} \%$ & 5.0 & 4.7 & \\
\hline Usage of steroids, $\$$ & 95.0 & 88.3 & 0.363 \\
\hline Dose of steroids, $\mathrm{mg}$ (ICR) & $6.0(5.0-12.5)$ & $6.0(4.0-9.0)$ & 0.284 \\
\hline $\begin{array}{l}\text { Uasege of } \\
\text { immosese }\end{array}$ & 50.0 & 50.9 & 0.904 \\
\hline Usage of SMX-TMP.X & 25.0 & 21.5 & 0.725 \\
\hline Uver distunction, \% & $0(0 / 20)$ & $0.5(1 / 213)$ & 0.759 \\
\hline Kidney distunction, $\%$ & $10.0(2 / 20)$ & $5.6(12 / 213)$ & 0.432 \\
\hline Positive anti-dSONA & $632(12 / 19)$ & 708 (148/209) & 0.485 \\
\hline 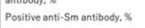 & $60.0(12 / 20)$ & $34.2(69 / 202)$ & 0.022 \\
\hline Positive ant-RNP antibody. $\mathbf{x}$ & $66.7(10 / 15)$ & $48.9(185 / 174)$ & 0.185 \\
\hline Positive ant-SSA antibody. $\%$ & $40.0(8 / 20)$ & $555(111 / 200)$ & 0.185 \\
\hline
\end{tabular}

Conclusions: $\mathrm{HCQ}$-induced hypersensitivity reaction typically presents with mild generalised eruption 1-4 weeks after starting HCQ. Restarting HCQ with the simple dose escalation regimen may be a practical option for patients with mild HCQinduced hypersensitivity reaction.

\section{REFERENCES}

[1] Yokogawa N, et al. Effects of Hydroxychloroquine in Patients With Cutaneous Lupus Erythematosus: A Multicenter, Double-Blind, Randomized, Parallel-Group Trial. Arthritis Rheumatol 2017;69:791-799.

[2] Tal $Y$, et al. Hydroxychloroquine desensitization, an effective method to overcome hypersensitivity-a multicenter experience. Lupus 2017;1961203317735185.

[3] Utsunomiya $\mathrm{M}$, et al. Optimal regimens of sulfamethoxazole-trimethoprim for chemoprophylaxis of Pneumocystis pneumonia in patients with sys temic rheumatic diseases: results from a non-blinded, randomized controlled trial. Arthritis Res Ther 2017;19:7.

Disclosure of Interest: None declared

DOI: 10.1136/annrheumdis-2018-eular.3206

\section{FRI0320 ANTIMALARIALS PROTECTIVE EFFECTS IN SYSTEMIC LUPUS ERYTHEMATOSUS IN CHINA}

F. Wang, W. Zhang, X. Feng, L. Sun. Department of Rheumatology and Immunology, the Affiliated Drum Tower Hospital of Nanjing University Medical School, Nanjing, China

Background: Hydroxychloroquine (HCQ) and chloroquine (CQ), both known as antimalarial drugs, have become fundamental therapeutic elements in systemic lupus erythematosus (SLE) in these decades. However, their specific benefits on organs or in a large cohort of Chinese SLE patients haven't been elucidated.

Objectives: This retrospective multicenter study sought to examine the potential protective roles of antimalarials in a large retrospect multicenter study of SLE patients in China.

Methods: Data were collected from 1372 patients' first and following hospitalizations during 1999 to 2009. Laboratory variables, medications, disease activity, organ involvements, and survival statuses were analysed according to antimalarial usage.

Results: Antimalarials improved the survival of SLE patients as an independent predictor (HR $0.503, p=0.000$ ). Using antimalarials protected patients with longer disease duration (>2 years), SLEDAI (>15) on admission, high SLEDAI (>10) on discharge, organ involvements (cardiopulmonary, gastrointestinal, renal, hematologic), anti-dsDNA positive, and no application of cyclophosphamide. In second hospitalisation, compared to non-users, SLE patients who had been treating with antimalarial drugs had lower levels of total cholesterol (TC) $(4.47(0.13) \mathrm{mmol} / \mathrm{L}$ vs $5.03(0.21) \mathrm{mmol} / \mathrm{L}, \mathrm{p}=0.027)$ and less chance of anaemia $(46.5 \%$ vs $58.2 \%$, $\mathrm{p}=0.033)$.
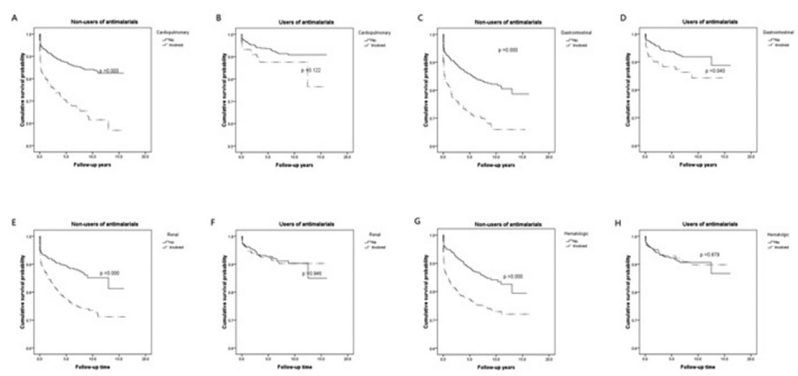

Conclusions: Using antimalarials benefits outcomes of SLE patients through multiple facets in Chinese patients.

Disclosure of Interest: None declared

DOI: 10.1136/annrheumdis-2018-eular.3073

\section{FRI0321 SURVIVAL RATE AND CAUSES OF WITHDRAWAL OF BELIMUMAB TREATMENT IN SLE IN A REAL LIFE SETTING}

F. Morello $^{1}$, F.R. Spinelli ${ }^{2}$, F. Ceccarelli ${ }^{2}$, L. Massaro ${ }^{2}$, L. Novelli ${ }^{2}$, F. Miranda ${ }^{2}$, C. Perricone ${ }^{2}$, S. Truglia ${ }^{2}$, C. Alessandri ${ }^{2}$, G. Valesini ${ }^{2}$, F. Conti ${ }^{2}$. ${ }^{1}$ Dipartimento di Medicina Interna e Specialità Mediche - Reumatologia, Sapienza Università di Roma, Rome, Italy, ${ }^{2}$ Dipartimento di Medicina Interna e Specialità Mediche Reumatologia, Sapienza Università di Roma, Rome, Italy, rome, Italy

Background: Systemic Lupus Erythematosus (SLE)is a chronic disease requiring long-term treatment. Even though immunosuppresive therapies improved the survival rate, a great percentage of SLE patients exhibit a persistently active disease, or disease flares. Belimumab (BLM), is currently the only biological drug 